ASM Sc. J., 13, 2020

https://doi.org/10.32802/asmscj.2020.sm26(2.4)

\title{
Analysis of Human's Attention Value in Gender and Age Category using Mindwave Mobile
}

\author{
Norasyimah Sahat ${ }^{1}$, Afishah Alias ${ }^{1 *}$ and Fouziah Md Yassin ${ }^{2}$ \\ ${ }^{1}$ Faculty of Applied Science and Technology, Universiti Tun Hussein Onn Malaysia, 84600 Muar \\ Johor, Malaysia \\ ${ }^{2}$ Faculty of Science and Natural Resources, Universiti Malaysia Sabah, 88400 Kota Kinabalu, \\ Sabah, Malaysia
}

\begin{abstract}
Brainwave-controlled robot using a Brain-Computer Interface (BCI) system was designed and studied to help people with disabilities, especially for people who suffer from motor disorders such as Amyotrophic Lateral Sclerosis (ALS). BCI is a communication system that does not depend on peripheral nerves and muscles. However, the integrated robot controlled by the human mind has less efficiency due to the different type of brainwaves generated by a user. The purpose of this paper is to analyze the human's attention value in age and gender category for improving the mind-controlled robot movement. An electroencephalography (EEG) device called Mindwave Mobile is used to get the attention value generated by humans in the different type of gender (male, female) and age category (children, teenager, adult). There are five aspects of thought movement generated by human which is forward (F), right (R), left (L), backward (B) and stop (S). For statistical analysis, the main effect of gender on the attention value was analyzed by using independent sample t-test and the main effect of age category was analyzed by oneway ANOVA test while the interaction effect between these two factors was analyzed by two-way ANOVA test. The results show that male has a higher attention value than female for every aspect of thought movement except for the left and backward movement. This is due to the different size of the frontal lobe in gender. For the age category, the teenager is more focused than children and adult while the adult has the lowest attention value in every aspect of the movement. From the analysis, only in the forward movement shows that there was a significantly different in gender difference while there was no significant difference in the main effect of age category and the interaction effect between the gender and age category on the attention value for every movement. By doing this research, the movement of a mindcontrolled robot can be improved by setting the threshold value depending on the gender and age category of the user.
\end{abstract}

Keywords: Attention value, Brain-Computer Interface, Brainwave, Mindwave Mobile

\section{INTRODUCTION}

Brain-Computer Interface (BCI) is a system that communicates between a human brain and physical devices by translating different patterns of the brain activity into command in real time (Sahat et. al., 2018). The objective of the BCI study is to restore vital functions to people who are highly disabled by various neuromuscular disorders and enhance the functioning of healthy individuals (Ramesh et. al., 2014).

In 1924, a German scientist called Hans Berger conducted a study on the human brain waves and recorded electrical brain activity and used the word electroencephalography (EEG) to describe brain electric potentials in humans. Brainwaves can be detected using sensors placed on the scalp (Millet, 2001). During the 1950's, EEG technology was

*Corresponding author's e-mail: afishah@uthm.edu.my 
increasingly applied in neurology, neurosurgery, and cognitive science. In 1964 Dr. Grew Walter connected electrodes directly to the motor areas of a subject's brain to record brain ac activity (Ali, 2012).

Recent advances in EEG technology have led to the development of cheaper and easier to set up products which use dry electrode-based EEG hardware, such as the Neurosky Mindwave Mobile. The Neurosky Mindwave headset was launched by Neurosky company and has been designed to identify and monitor electric signals generated by neural activity in the brain. It complements the Neurosky Mindset headset, released in 2009, which has been used to research ADHD, Alzheimer's, and Cognitive Stress (Mostow et. al., 2011).

Neurosky Mindwave Mobile is a device for monitoring electrical signals generated by neural activity in the brain. These tools can read brain waves and apply them to computers or to mobile platforms that are connected via Bluetooth (Devi et al., 2014). It is low cost, accessible and simple to operate compared to traditional EEG devices. It also compatible with PCs, Mac and mobile devices like iPhone, iPad, iOS or mobile operating systems like Android or iOS (Robbins \& Stonehill, 2014).

This device consists of an adjustable headband, a sensor tip (arm) \& an ear clip (the second sensor), the battery area \& the power switch. Inside the sensor arm is the ThinkGear AM chipset (EEG sensor) along with dry electrodes and a specially designed electronic circuit perceives the brain signals, filter out the noise and the muscle movement, converting to digital power. Mindwave Mobile also has an electromyography (EMG) sensor which also detects the blinking movement (Akila et. al., 2015). Neurosky Mindwave Mobile can measure raw signal, EEG power spectrum, eSense meters for Attention and Meditation, eSense blink detection and poor signal quality. The formula for converting raw values to voltage for Mindwave Mobile device is: [raw Value*(1.8/4096)] / 2000 (Obermaier et. al., 2001)

This is due to a 2000x gain, 4096 value range, and $1.8 \mathrm{~V}$ input voltage. eSense Attention meter is used to obtain the digital value of attention produced by the Mindwave Mobile user. Disturbance, drifting thoughts, less focus or anxiety can lower the level of the person's attention. Table 1 shows five categories of attention level produced by the Mindwave
Mobile user. The attention level at neutral was considered as normal concentration level. The attention levels at less and poor were lower than normal attention level. The attention level higher than the neutral level indicates the person has a higher concentration level now. People with "great attention" level which range between 80 and 100 have very high concentration level at that time (Yassin et. al., 2017).

Table 1. Five categories of attention levels (Neurosky, 2009)

\begin{tabular}{|c|c|}
\hline Attention level & Category \\
\hline $80-100$ & Great attention \\
\hline $60-79$ & Good attention \\
\hline $40-59$ & Neutral \\
\hline $20-39$ & Less attention \\
\hline $1-19$ & Poor attention \\
\hline
\end{tabular}

In this paper, Neurosky Mindwave Mobile is used as a brainwave sensor to detect and analyze the human's attention value which is related to the beta wave produced by the human in different type of gender (male and female) and age category (children, teenager and adult) and investigate the effects of these two factor to the attention value in every aspect of thought movement.

\section{MATERIALS AND METHODS}

\section{A. Experimental Setup}

Thirty (30) of respondents were selected for the analysis of human's attention value using Neurosky Mindwave Mobile. There were 10 children (6-12 years old), 10 teenagers (18-25 years old) and 10 adults (30 years and over). Each subject had to think five components of thought movement which is forward (F), right (R), left (L), backward (B) and stop (S). The data was collected and recorded within one minute for every aspect of the movement. Figure 1 shows the flow diagram of the experiment conducted to an individual for each type of gender and age category. 


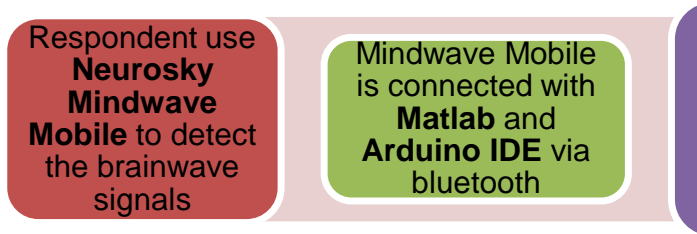

The thought
movement is divided
into five components
namely forward (F),
right (R), left (L),
backward (B) and
stop (S).

\section{The test is} conducted for one minute for every aspect of thought movement
The user's attention value wil be recorded and measured.

Figure 1. Experimental flow diagram of Mindwave Mobile users (Yassin et al., 2018)

\section{B. Analysis Procedure}

There are two methods of measuring human's attention value produced by the user in each type of gender and age

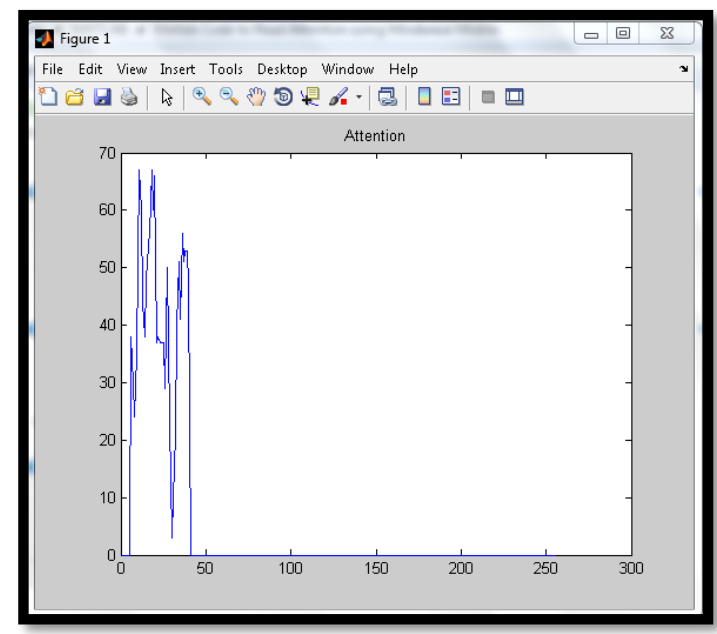

Figure 2. The graphical form obtained in MATLAB software

For data analysis, the main effect of gender was analyzed by independent sample t-test while the effect of the age category on attention value was analyzed by one-way ANOVA tests. The interaction effect of gender and age category on the attention value produced by the user in every aspect of the movement were analyzed by two-way ANOVA analysis. ANOVA is a collection of statistical models used to analyze the differences between group means and their associated procedures. It consists of the null hypothesis and alternative hypothesis regarding the differences or effects that occur in the population. The null hypothesis is the statement that needs to prove while the alternative hypothesis is the opposite of null (Tomari et. al., 2015). category. First, the MATLAB software (Figure 2) is used to obtain the brainwave analog or graphical form. Secondly, the Arduino Integrated Development Environment (IDE) software (Figure 3) is used to get the attention of the user in the digital form.
PoorQuality: 0 Attention: 100 Time since last packet: 997 PoorQuality: 0 Attention: 100 Time since last packet: 987 PoorQuality: 0 Attention: 97 Time since last packet: 998 PoorQuality: 0 Attention: 100 Time since last packet: 994 PoorQuality: 0 Attention: 100 Time since last packet: 1000 PoorQuality: 0 Attention: 100 Time since last packet: 994 PoorQuality: 0 Attention: 100 Time since last packet: 995 PoorQuality: 0 Attention: 100 Time since last packet: 997 PoorQuality: 0 Attention: 93 Time since last packet: 1000 PoorQuality: 0 Attention: 90 Time since last packet: 993 PoorQuality: 0 Attention: 96 Time since last packet: 995 PoorQuality: 0 Attention: 100 Time since last packet: 1003 PoorQuality: 0 Attention: 100 Time since last packet: 991 PoorQuality: 0 Attention: 93 Time since last packet: 996 PoorQuality: 0 Attention: 88 Time since last packet: 998 PoorQuality: 0 Attention: 67 Time since last packet: 997 PoorQuality: 0 Attention: 57 Time since last packet: 993 PoorQuality: 0 Attention: 56 Time since last packet: 998 PoorQuality: 0 Attention: 51 Time since last packet: 999

Figure 3. Attention value obtained in the serial monitor using Arduino IDE software

\section{RESULTS AND DISCUSSIONS}

\section{A. Effect of Gender on the Attention Value of Human in Every Aspect of the Movement}

The average of the attention value of the subjects based on gender differences ( 15 males and 15 females) has been calculated and can be shown in Figure 4. Some researcher said that there might be differences between male and females in learning and thinking abilities. Research by Chief and Shallcross proved that males and females have different paying attention abilities (Chief \& Shallcross, 1992). Figure 
4 shows that males have a higher attention level compared to the females in every aspect of the movement except for the left and backward movement. This is different from the investigation study by Chua Yan Piaw. He conducted some experiments to study the relationship between thinking styles and the ability to pay attention to Malaysian male and female student teachers. The results found out that female subjects showed better ability to pay attention compared to the male subjects (Piaw, 2014).

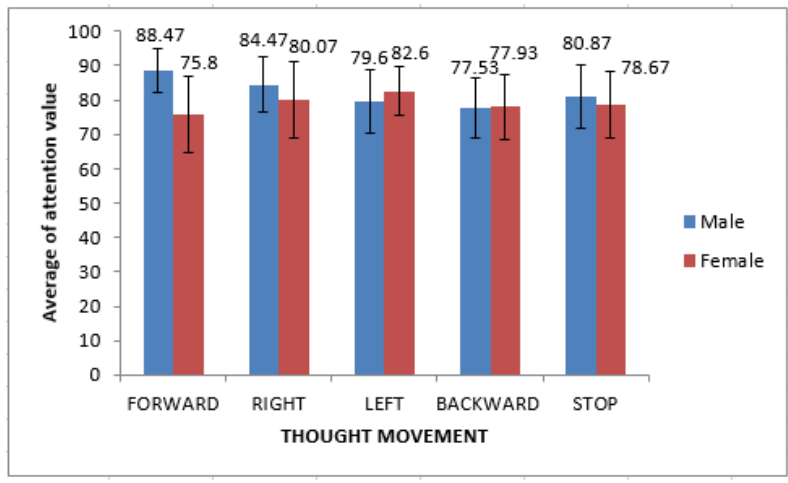

Figure 4: Average of the attention value based on the gender differences

An independent-samples t-test was conducted to compare attention value for every aspect of movement between males and females with the level significance, $\alpha=0.05$. Attention level for each level of gender were normally distributed, as assessed by Shapiro-Wilk test ( $\mathrm{p}>.05)$. Homogeneity of variance as accessed by the Levene's test is not met only for forward movement because the sig. value which is 0.034 is less than alpha. Therefore, the data results associated with the "Equal variances not assumed" is used.
Result in Table 2 shows that attention value in forward movement is the only component which yielded a significant difference between male and female participants. In this case, the attention value for male is higher than female for the forward movement [forward mean score: male $=88.47$, female $=75.80$; mean differences $=12.67, \mathrm{p}<.05]$. However, there were no significant differences between male and female participants for another four aspects of the thought movement.

Table 2. Comparison between male and female participants on attention value for five aspects of thought movement

\begin{tabular}{ccccc}
\hline Dependent variable & Levene's test & $\begin{array}{c}\text { Mean } \\
\text { Difference }\end{array}$ & t & Sig. \\
\hline Forward (F) & 0.03 & 12.67 & 2.14 & $0.04^{*}$ \\
Right (R) & 0.29 & 4.40 & 0.69 & 0.50 \\
Left (L) & 0.14 & -3.00 & -0.56 & 0.58 \\
Backward (B) & 0.43 & -0.40 & -0.07 & 0.95 \\
Stop (S) & 0.80 & 2.20 & 0.36 & 0.72 \\
\hline
\end{tabular}

Note: * significant at $\mathrm{p}<.05$

\section{B. Effect of Age Category on the Attention Value of Human in Every Aspect of the Movement}

Figure 5 shows that a teenager has the highest attention value when doing right and backward movement and has the same attention value with the children for the left movement. While for the forward and stop movement, children category has the highest attention value among the group and the adult has the lowest attention value for all aspect of the movement. There are several factors

that can affect the attention value produced by the human in each category of age such as surrounding and disease factors. The previous study shows that brain disease such as epilepsy and attention deficit disorder which usually happen to children also can lead to less attention (Ardeshiri et. al., 
2006).

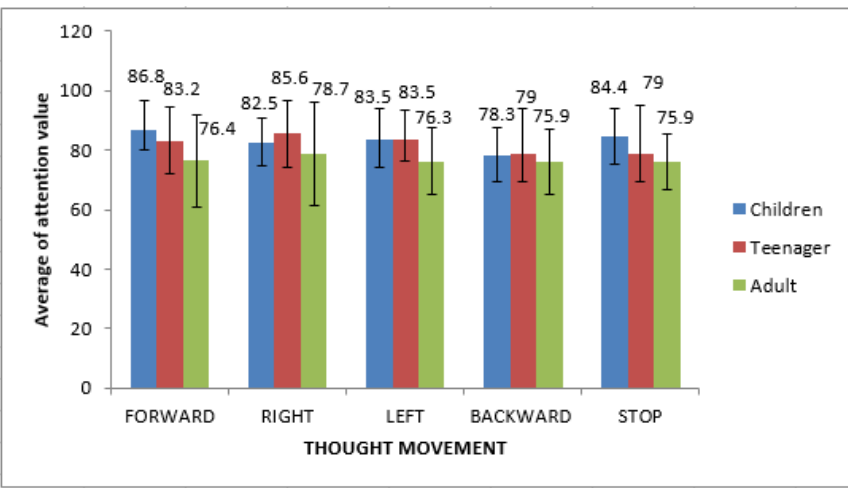

Figure 5: Average of the attention value based on the age category

The data in Table 3 reveals differences between the three categories of age across the five type of thought movement for the attention value. Shapiro-Wilk test is used to test the assumption of normality and Levene's test for the assumption of homogeneity of variance. Based on the result in SPSS, the data were normally distributed as the $\mathrm{p}$ of the Shapiro-Wilk test id higher than alpha level. For the homogeneity of variance, result show that the $\mathrm{F}$ value for Levene's test for the right and stop movement is 5.84 and 3.95 with a sig. (p) value of .01 and .03 which is less than alpha of .05, concluded that the assumption of homogeneity of variance is not met while for other three movements, the sig. value is greater than alpha level concludes that there is not a significant difference between the group's variance.

Using an alpha level of 0.05, one-way ANOVA test was used to determine if there is a significant difference in attention value of participant among age category which is children, teenager and adult. The result shows that there was no significant difference between age categories on the attention value for every aspect of the movement.

Table 3. Effect of age category on attention value for every aspect of the movement

\begin{tabular}{|c|c|c|c|c|}
\hline \multirow{2}{*}{$\begin{array}{c}\text { Dependent } \\
\text { variable }\end{array}$} & \multicolumn{2}{|c|}{ Homogeneity of variance } & \multicolumn{2}{|c|}{ ANOVA } \\
\hline & Levene statistic & Sig. & $\mathbf{F}$ & Sig. \\
\hline & 0.71 & 0.50 & 0.94 & 0.40 \\
\hline Right (R) & 5.84 & 0.01 & 0.38 & 0.69 \\
\hline Left (L) & 0.10 & 0.91 & 0.80 & 0.46 \\
\hline Backward (B) & 2.07 & 0.15 & 0.10 & 0.91 \\
\hline Stop (S) & 3.95 & 0.03 & 0.66 & 0.53 \\
\hline
\end{tabular}

\section{The Interaction Between Gender and Age} Category with Respect to the Attention Value Produced by the Human in Every Aspect of the Movement
Table 4 indicates the results of two-way ANOVA tests for the interaction effect between two factors which is gender and age category on the attention value in every aspect of movement. The result shows that there were no significance differences between gender and age category with respect to the attention value for every aspect of the movement. 
Table 4. Effects of gender and age category on the attention value for every aspect of movement

\begin{tabular}{|c|c|c|c|c|}
\hline Factor & Type of movement & df1, df2 & $\mathbf{F}$ & Sig. \\
\hline \multirow{5}{*}{ Gender } & Forward (F) & 1,24 & $4 \cdot 37$ & $0.05^{*}$ \\
\hline & Right (R) & 1,24 & 0.42 & 0.52 \\
\hline & Left (L) & 1,24 & 0.28 & 0.60 \\
\hline & Backward (B) & 1,24 & 0.004 & 0.95 \\
\hline & Stop (S) & 1,24 & 0.12 & 0.73 \\
\hline \multirow{5}{*}{ Age } & Forward (F) & 2,24 & 1.01 & 0.38 \\
\hline & Right (R) & 2,24 & 0.34 & 0.71 \\
\hline & Left (L) & 2,24 & 0.73 & 0.50 \\
\hline & Backward (B) & 2,24 & 0.09 & 0.92 \\
\hline & Stop (S) & 2,24 & 0.62 & 0.55 \\
\hline \multirow{5}{*}{ Gender*Age } & Forward (F) & 2,24 & 0.30 & 0.74 \\
\hline & Right (R) & 2,24 & 0.08 & 0.92 \\
\hline & Left (L) & 2,24 & 0.11 & 0.89 \\
\hline & Backward (B) & 2,24 & 0.14 & 0.87 \\
\hline & Stop (S) & 2,24 & 0.63 & 0.54 \\
\hline
\end{tabular}

Note: *significant at $\mathrm{p}<.05$

\section{CONCLUSION}

Based on the findings obtained through the experiment carried out revealed that the gender and age category could affect the attention level produced by humans. Male is easier to focus compared to the female for every aspect of the thought movement except for the left and backward movement. The teenager has the highest attention value when doing right and backward movement. While for the forward and stop movement, children category has the highest attention value and the adult has the lowest attention value for all aspect of the movement. For statistical analysis, only in the forward movement shows that there was a significantly different between male and female while there was no significant difference in the effect of age category and the interaction effect between the gender and age category with respect to the attention value for every movement.

\section{ACKNOWLEDGMENT}

The author is grateful to Universiti Tun Hussein Onn Malaysia and Universiti Malaysia Sabah for providing relevant instruments in conducting this research. This work was funded by Research Management Centre, Universiti Tun Hussein Onn Malaysia under Tier 1 (Ho66) and GPPS (Ho53) Research Grant. 


\section{REFERENCES}

Akila, M., Sekar, K. S., Suresh, A. 2015, Smart braincontrolled wheelchair and devices based on EEG in low cost for disabled person. International Journal of Computers Communication Networks and Circuit Systems, 1(1), pp. 291-298

Ali, M. Z. 2012, EEG-based assessment of driver's cognitive response in virtual traffic light environment. Lamar University-Beaumont.

Ardershiri, A., Wenger, E., Holtmannspotter, M., \& Winkler, P. A. 2006, Surgery of the anterior part of the frontal lobe and of the central region: normative morphometric data based on magnetic resonance imaging, Neurosurgical review, 29(4), pp. 313-321

Chief, L. \& Shallcross, D. J. 1992, Effect of the assumed boundary in the solving of the nine-dot problem on a sample of Chinese and American students 6-18 years old Journal of Creative Behaviour, 26(1), pp. 53-66

Devi, M. A., Sharmila, R., Saranya, V., 2014, January, "Hybrid brain computer interface in wheelchair using voice recognition sensors, International Conference on Computer Communication and Informatics (ICCI), pp. $1-5$

Millet, D. 2001, Hans Berger: From psychic energy to the EEG, Perspective in biology and medicine, vol. 44, no. 4, pp. 522-542

Mostow, J., Chang, K. M., Nelson, J., 2011, June, “Toward Exploiting EEG input in a reading tutor", In International Conference on Artificial Intelligence in Education (pp. 230-237). Springer, Berlin, Heidelberg.

Neurosky Inc (Ed). 2009, Neurosky eSense TM Meters and
Detection of Mental State.

Obermaier, B., Neuper, C., Guger, C., Pfurtscheller, 2001, "Information transfer rate in a five classes braincomputer rate in a five-classes brain-computer interface”, IEEE Transactions on neural systems and rehabilitation engineering, 9(3), pp. 283-288

Piaw, C.Y. 2014, Effects of Gender and Thinking Style on Student's Creative Thinking Ability. Procedia-Social and Behavioral Sciences, 116, pp.5135-5139.

Ramesh, S., Krishna, M., Nakirekanti, M. 2014, Brain Computer Interface System for Mind Controlled Robot using Bluetooth. International Journal of Computer Applications, vol. 104, no. 15, pp. 20-23

Robbins, R. \& Stonehill, M. 2014, Investigating the Neurosky Mindwave EEG Headset. Transport Research Foundation, 1(28), pp. 14-20

Sahat, N., Alias, A., Yassin, M. D. F. 2018, Brainwave Analysis for Robot Movement Depending on Age and Sex Differences. International Journal of Engineering \& Technology, 7(4.30), pp. 276-280

Tomari, R., Hassan, R. R. A., Zakaria, W. N. W., \& Ngadengon, R. 2015, Analysis of Optimal Brainwave Concentration Model for Wheelchair Input Interface. Procedia Computer Science, 76, pp. 336-341

Yassin, M. D. F., Apin, D., Abd. Rahman, A. B., Alias, A. 2017, Multi-mode Brainwave Controller. Advanced Science Letters, 23(11), pp. 11508-11511

Yassin, M. D. F., Sahat, N., Chin, S. N., Alias, A., 2018, The brainwave analysis for robot movement using one electrode. Academic Journal of Science, 8(1), pp. 15-22 